\title{
Mice (Mus musculus) do not perceive emergent Gestalt
}

\author{
Kazuhiro Goto ${ }^{1} \&$ Hikari Watanabe ${ }^{1}$ \\ ${ }^{1}$ Department of Human Psychology, Sagami Women's University
}

Postprint of manuscript accepted for publication at Journal of Experimental Psychology: Animal Learning and Cognition on March 9, 2021. (C2021, American Psychological Association. This paper is not the copy of record and may not exactly replicate the final, authoritative version of the article. The final article is available via its DOI: 10.1037/xan0000291.

We have previously demonstrated that chimpanzees, similar to humans, can discriminate the orientations of a diagonal line better when lines are presented in redundant contexts than alone. In the present study, we examined whether the same redundant context facilitated diagonalorientation discrimination in mice. Mice were presented one of three simultaneous, diagonalorientation discrimination tasks: (a) presented alone, (b) presented with the context that resulted in emergent configurations in chimpanzees and humans, and (c) presented with the context not resulting in emergent configurations in chimpanzees or humans. In contrast to the facilitative effect of congruent context in chimpanzees and humans, the identical context did not facilitate the discrimination of the diagonal orientation in mice. This finding suggests that mice, unlike chimpanzees and humans, do not perceive emergent Gestalt.

Keywords: Gestalt, wholes, parts, rodent, visual percetion Word count: 3,831

At times, the perception of the whole has characteristics that are not present among its component parts. Experiments showing that the discrimination of a line's slope becomes easier when the lines are embedded within a well-structured context provides empirical evidence of such Gestalt properties. As shown in Figure 1, humans discriminate a diagonal line from another diagonal line with a 90-degree difference more quickly and more accurately when the lines are presented with redundant contextual information about each diagonal (Pomerantz, 2003; Pomerantz \& Portillo, 2011;

Kazuhiro Goto https://orcid.org/0000-0003-0548-3147. This work was funded by a Grant-in-Aid for Scientific Research (C) (20K03485) and by a Grant-in-Aid for Scientific Research on Innovative Areas (20H05017) from the Ministry of Education, Culture, Sports, Science, and Technology of Japan to Kazuhiro Goto. The authors thank Kaori Uyama for her assistance with animal maintenance and in conducting experiments and Yuya Hataji for his thoughtful comments on the manuscript. The data are available at https://osf.io/53zhg/.Correspondence concerning this article should be addressed to Kazuhiro Goto, Department of Human Psychology, Sagami Women's University, 2-1-1 Bunkyo Minami-Ku Sagami Kanagawa, 252-0307, Japan. Email:kazuhiro.goto@gmail.com

Correspondence concerning this article should be addressed to Kazuhiro Goto, 2-1-1 Bunkyo, Minami-ku, Sagamihara, Kanagawa, 252-0383. E-mail: kazuhiro.goto@gmail.com
Pomerantz, Sager, \& Stoever, 1977). This facilitation effect, known as configural superiority effect, might occur because the percept of wholes by humans differs from the summation of its component parts. Novel Gestalts, such as openness and closure, emerge when stimuli are presented within a congruent context. The congruency of the context is defined by empirically confirming that the inclusion of redundant contexts facilitates discrimination.

We have previously compared chimpanzees with humans and examined whether nonhuman primates perceive emergent Gestalts identically to humans (Goto, Imura, \& Tomonaga, 2012). Chimpanzees and humans searched for a target line that differed from three other distractor lines with a 90degree difference in direction. There were three context conditions: (a) presented alone, (b) presented with the identical context that resulted in emergent Gestalts in humans (congruent context), or (c) presented with the identical context that did not result in emergent Gestalts in humans (incongruent context). Similar to humans, Chimpanzees found a target faster and more accurately when the lines were presented in a congruent context than presented alone. Similarly, neither species demonstrated any target localization facilitation when the line orientations were presented in an incongruent context. These results suggest that chimpanzees perceive the same emergent Gestalts as humans.

We have also examined whether pigeons and crows perceive emergent Gestalts using the same task and stimuli as those 

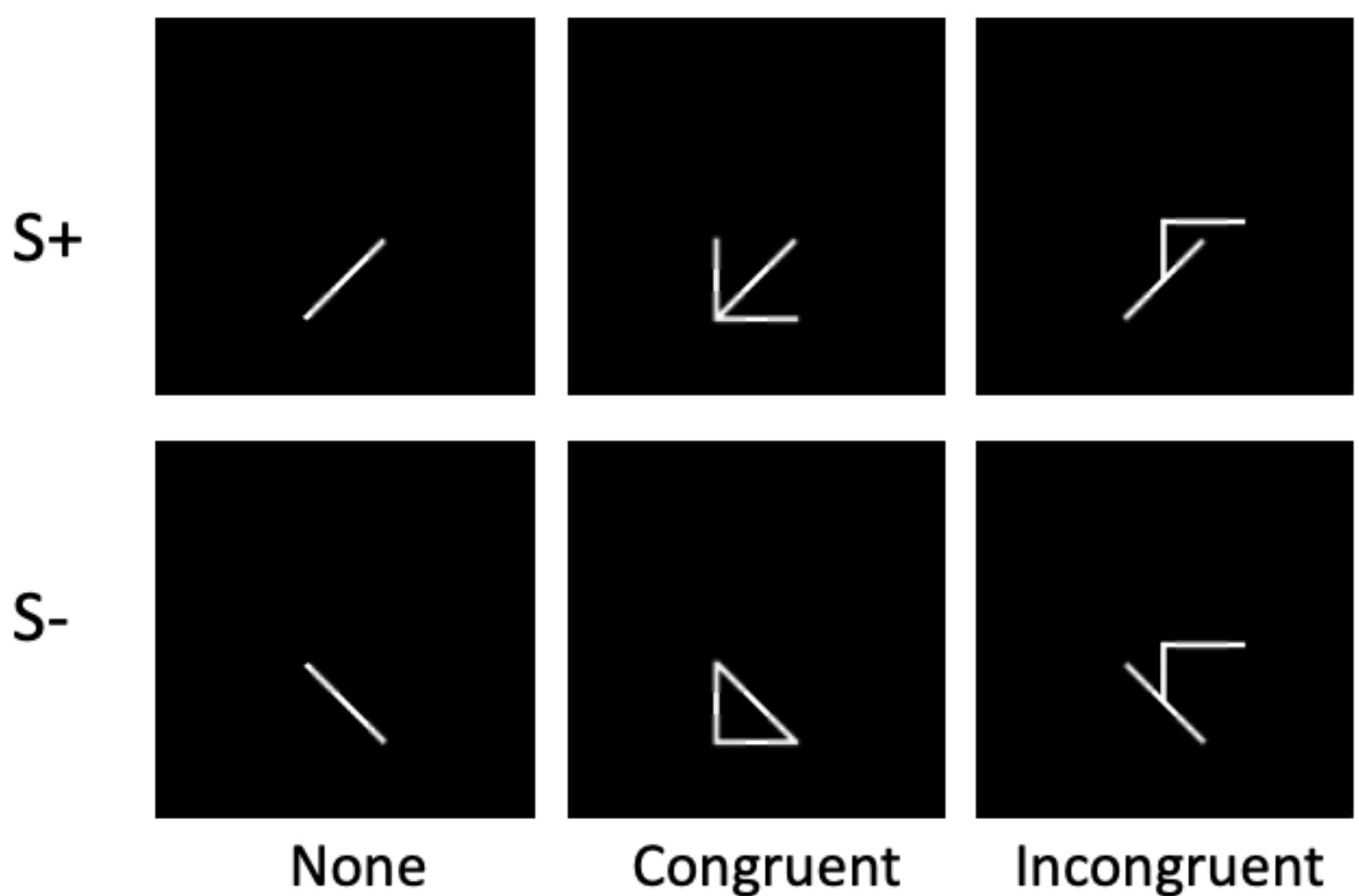

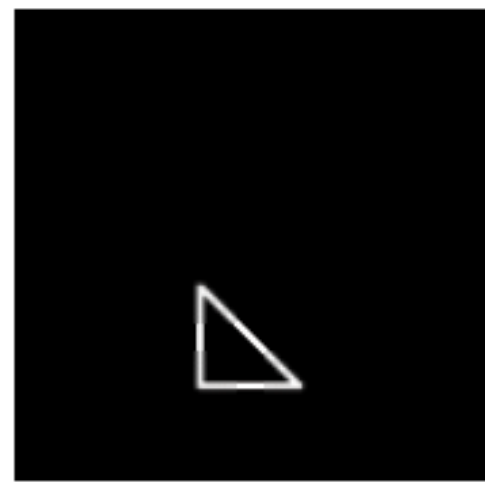

Congruent

\section{Context}

Figure 1. Stimulus pairs used in the present study. Each stimulus pair consisted of S+ and S-. Choosing S+ was correct, whereas the choosing S- was considered incorrect. The congruent context facilitated target localization for humans and chimpanzees despite the redundancy between the context and the discrimination of the diagonal directions (Goto, Imura, \& Tomonaga, 2012). In contrast, the incongruent context disrupted target localization for humans and chimpanzees.

used in our study of chimpanzee (Goto \& Watanabe, 2020). Pigeons and crows localized a target more slowly when a line was presented within a congruent context than presented alone, suggesting that birds do not perceive the same emergent Gestalts as chimpanzees and humans. Similarly, Donis and Heinemann (1993) demonstrated that such redundant contexts disrupt discrimination learning in pigeons using a successive discrimination procedure. Donis, Chase, and Heinemann (2005) further tested pigeons with varieties of stimulus sets and concluded that redundant contexts always disrupted discrimination. Kelly and Cook (2003) also compared pigeons with humans by using a same-or-different discrimination procedure and reported that the disruptive effect of redundant contexts on diagonal-orientation discrimination in pigeons contrasted with the facilitative effect of redundant contexts for humans.
It has been considered that such differential effects of redundant contexts between these two taxa are related to the functional difference between birds and mammals (Goto \& Watanabe, 2020; Lea, Goto, Osthaus, \& Ryan, 2006; Qadri \& Cook, 2015). However, several other comparative studies have reported contradictory results. For example, Neiworth et al. (2014) found that cotton-top tamarins discriminated diagonal lines less well when presented within a congruent context than presented alone. Talpos, de-Wit, Olley, Riordan, and Steckler (2016) also reported a similar disruptive effect of congruent context in rats, implying that the perception of emergent Gestalts differs among mammals.

The present study aimed to examine the effect of redundant context in yet another mammalian species. We chose mice as experimental subjects to examine whether the findings of Talpos, de-Wit, Olley, Riordan, and Steckler (2016) could 
be generalized to other rodent species. Talpos, de-Wit, Olley, Riordan, and Steckler (2016) trained two groups of rats to discriminate the pair of stimuli shown in Figure 1. The rats in the part discrimination group were trained to only discriminate diagonal lines, whereas the rats in the whole discrimination group were trained to discriminate diagonal lines presented within the congruent context. The results indicated that the part discrimination group improved accuracy as training proceeded, whereas the whole discrimination group did not show accuracy above chance, suggesting that rats do not perceive emergent Gestalt. It is important to note that the experimental design of Talpos, de-Wit, Olley, Riordan, and Steckler (2016) differed from other studies in two critical aspects. First, they used a between-subject comparison, whereas a within-subject comparison was used in other studies. The weakness of their study was that the rats failed to discriminate slopes embedded within a congruent context. This failure may have been due to some procedural problems that may have applied only to the whole discrimination group. Therefore, it is essential to examine whether the results of Talpos et al. can be replicated using a within-subject design. Second, Pomerantz and Portillo (2011) pointed out the importance of including a control context. Therefore, our previous studies included incongruent contexts that did not generate emergent Gestalts for humans (Goto, Imura, \& Tomonaga, 2012; Goto \& Watanabe, 2020). In contrast, Talpos, de-Wit, Olley, Riordan, and Steckler (2016) did not include such a control context in their experimental design. The present study was designed to compare the effects of congruent and incongruent contexts.

\section{Methods}

\section{Subjects}

We used six male C57BL/6N mice purchased from Japan SLC at 8 weeks of age. They were kept in a cage $(182 \times$ $260 \times 128 \mathrm{~mm}$ ) filled with paper bedding (Shepherd Specialty Papers, Alpha-Dri) as a group, under a day-night cycle (12-hr light:12-hr dark; lights on: 6:00 a.m. to 6:00 p.m.). The mice were acclimated to the rearing room for 2 weeks before the experiment was started. The animals were kept on a restricted diet after starting the experiment. They weighed 24.4g (range: 23.5-26.5) and 20.7 g (range: 19.5-22.5) preand post-dietary restrictions, respectively. The weight was maintained by supplementary feeding (typically $1.75 \mathrm{~g}$ per animal a day) in addition to the food reinforcers in daily testing sessions. Water was freely available in the housing cages. The experiment was conducted during the light phase. This study was conducted according to the guidelines published by the Japan Society for Animal Psychology. The study was approved by the Animal Care and Use Committee of Sagami Women's University (Approval No. 2017-05).

\section{Apparatus}

As shown in Figure 2, the test was conducted in an operant conditioning chamber housed within a sound-attenuating box (Med Associates, ENV-022V). The chamber (Med Associates, ENV-307W) consisted of a metal frame, transparent Perspex walls, and a stainless-steel grid floor, with internal dimensions of $21.6 \mathrm{~cm}$ length $\times 18 \mathrm{~cm}$ wide $\times 17.8 \mathrm{~cm}$ height . A pellet receptacle (magazine) was placed at the back wall's center, and a pellet dispenser situated outside the box delivered 20-mg pellets (Bioserve, F0071-J50). A 1.0-A house light and tone generator (Med Associates, ENV-324W) were fitted to the chamber's back wall. The magazine was illuminated by an LED light and equipped with photocell detectors (Med Associates, ENV-303HDW) for sensing the mouse's head entry into the magazine. The chamber's front wall was a 10.4-inch touchscreen monitor $(1,024 \times 768$ pixels $)$ equipped with an infrared sensor (K-limbic systems). A black removable screen mask was placed over the screen. The mask had two response windows $(7.5 \mathrm{~cm} \times 7.5 \mathrm{~cm})$ through which the mouse could make a nose-poke at the screen. A bar fence, which helped prevent irrelevant or ineffective touchscreen activation, was positioned in front of the touchscreen. The two response windows in which stimuli were displayed could be accessed above the bar fence $2 \mathrm{~cm}$ from the chamber's floor. Control of the house light, magazine light, dispenser, and detection of the head entry to the magazine was conducted with a DIO board (Velleman, K8055) connected to a computer (Lenovo, ThinkPad T440p) situated near the chamber. All aspects of the system were controlled by custom software written in Visual Basic 6 (Microsoft).

\section{Stimuli}

We used three stimulus pairs that differed with regards to context (see Figure 1). In the no-context pair, the positive stimulus $(\mathrm{S}+)$ was a diagonal line that was inclined 45 degrees from the horizontal, whereas the negative stimulus (S-) was a diagonal line that differed 90 degrees in the counterclockwise direction from $\mathrm{S}+$. In the congruent-context pair, identical L-shaped contexts were added to S+ and S- stimuli, resulting in an arrow (open figure) and a triangle (closed figure). In the incongruent-context pair, L-shaped contexts were added to $\mathrm{S}+$ and $\mathrm{S}$ - stimuli such that both resulted in open figures. The line width of the figures was $1 \mathrm{~mm}$, and the size of the stimuli was approximately $4 \mathrm{~cm}^{2}$. One stimulus was presented within each window, such that the bottom edge of the stimulus was $1.8 \mathrm{~cm}$ above the bottom edge of the window. 
A

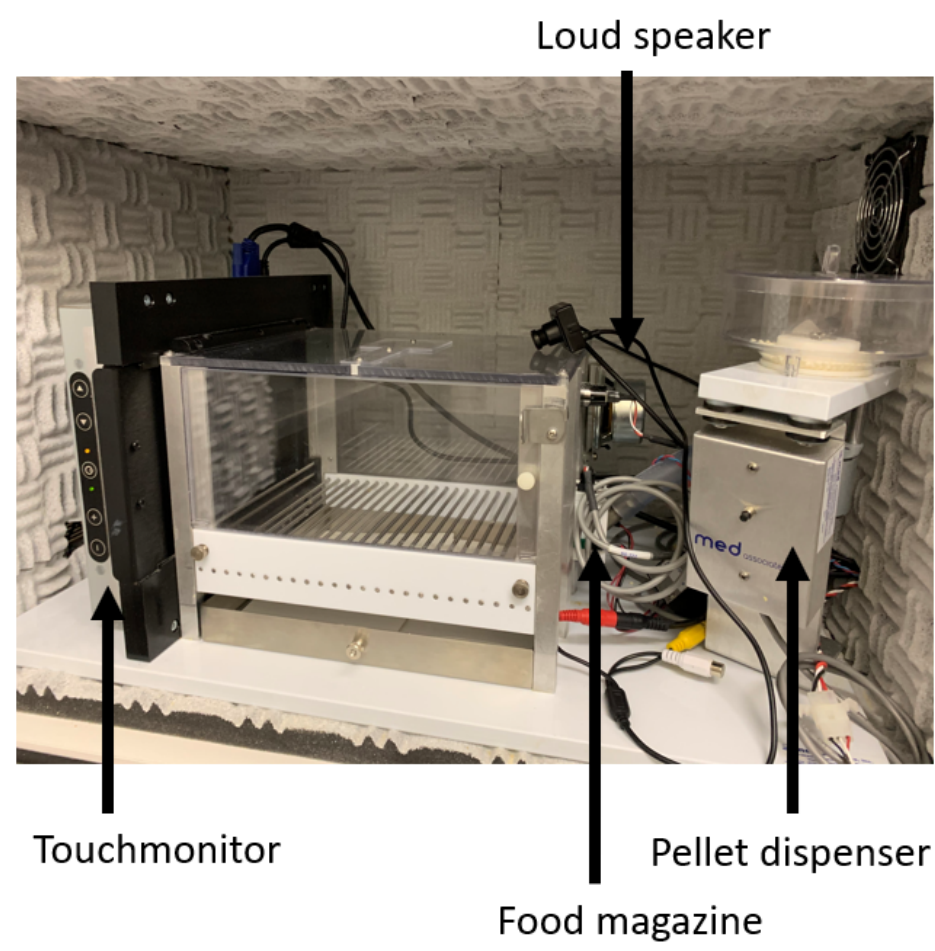

B

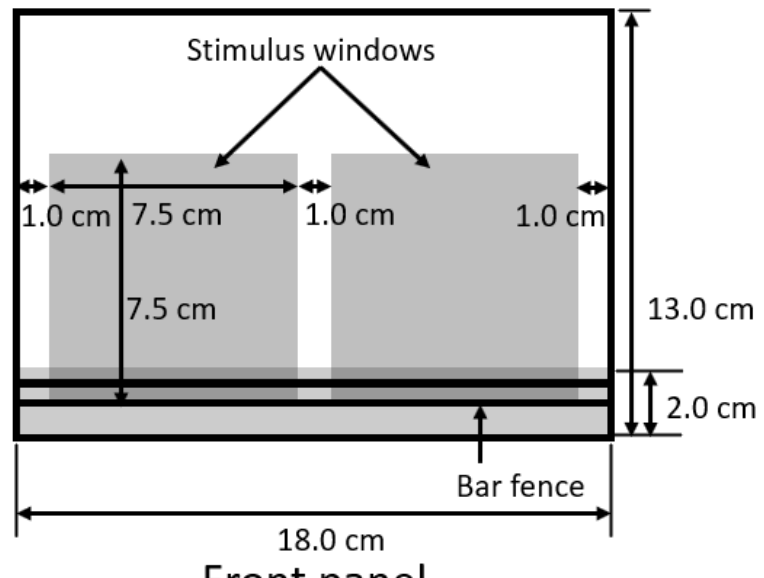

Front panel

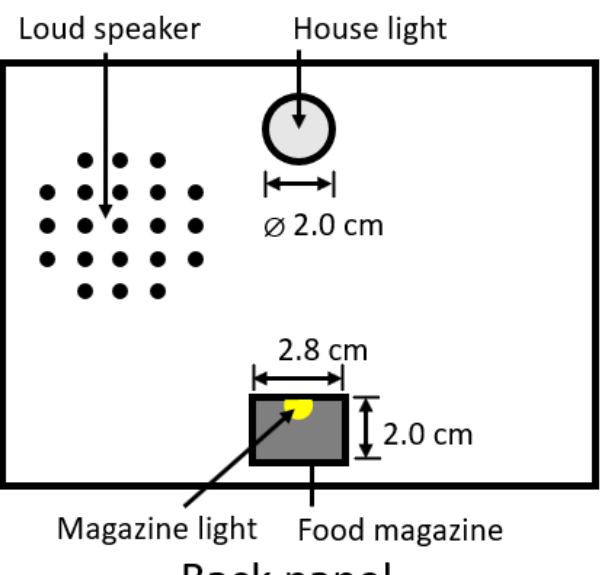

Figure 2. Touchscreen operant-conditioning chamber. (A) The touchscreen was mounted on the front panel. A metal frame was placed in front of the monitor. The frame has two windows in which stimuli were presented. A loudspeaker mounted on the rear panel provided feedback sound for correct responses. Pellet dispenser delivered a 20-mg pellet into a food magazine. (B) An illustration of the front and back panels of the operant-conditioning chamber.

\section{Procedure}

Mice were habituated to the chamber and given magazine training before training them to nose-touch a white circle (2.0 $\mathrm{cm}$ diameter) presented on the monitor's left window. After the acquisition of nose-touching responses, a stimulus was randomly presented on either the left or the right window in each trial. Then, a self-start signal was introduced. Each trial began with the magazine light blinking at 250-ms intervals. The mice's head entry to the magazine initiated the trial. Details of the training protocols are described in our previous report (Goto \& Hataji, 2020).

Figure 3 illustrates a discrimination trial sequence. Each trial began with the blinking of the magazine's light. The magazine light was turned off when the mouse entered its head into the magazine, and two stimuli were presented on the monitor, one in each stimulus window. The mouse turned around after the start response when it was approximately 10 $\mathrm{cm}$ from the monitor. The mouse then approached the monitor and appeared to choose one of the two stimuli when it was approximately $2.5 \mathrm{~cm}$ from the monitor. One of the highest, behaviorally-measured, visual acuities in mice is reported as .49 cycles per degree (Prusky, West, \& Douglas, 2000). This acuity would correspond to $0.89 \mathrm{~mm}$ if the mouse were to view the screen from $2.5 \mathrm{~cm}$ and $3.56 \mathrm{~mm}$ if to view the screen from a $10-\mathrm{cm}$ distance. Therefore, mice's visualspatial resolution was adequate to discriminate the stimuli. Because the stimulus size was small, a nose touch anywhere on the stimulus window was considered a valid response. Initially, a single touch was considered a choice response. The choice response was then increased to two consecutive touches on the same stimulus window from Session 31 
and further increased from Session 36 to four consecutive touches. This was done to increase the attention of mice to the stimuli and facilitate discrimination learning. Nose touches to $\mathrm{S}+$ stimuli were considered correct responses and they were reinforced by a $20-\mathrm{mg}$ pellet, 1 -s chime, and a 5$\mathrm{s}$ illumination of the magazine. In contrast, nose touches to S- stimuli were considered incorrect responses, and the trial ended without reinforcement and a 5-s blackout. The trial was repeated after an incorrect choice until the correct choice was made. These correction trials were excluded from the subsequent analysis. The intertrial interval was $20 \mathrm{~s}$. Each daily session consisted of 30 randomly mixed trials after excluding correction trials. These trials were evenly divided among the three stimulus pairs. The S+ stimuli were presented equally often on the left and right sides with equal probability. The training was continued for 5 days a week until 90 sessions were completed. Therefore, the training consisted of 2,700 trials. A video recording of this mouse's behavior is available at https://osf.io/hvk4c/.

\section{Statistical Analysis}

The primary measure of interest in this study was the proportion of correct responses under each context type. The mean proportion of correct responses was calculated for every 10-session blocks and each subject's context type. A two-way repeated-measures analysis of variance (ANOVA) with context type and session block as within-subject factors was conducted. We reported generalized eta-squared $\left(\eta g^{2}\right.$ : Olejnik \& Algina, 2003) as a measure of effect size. Response times were analyzed by first recording the time from the stimulus onset to the first of consecutive touch responses as the response time. Response times in the correct trials were log-transformed, and the mean of each 10-session block was calculated as the context type for each subject (all means were then transformed back to milliseconds by the exponential function). A two-way repeated-measures ANOVA with the context type and session blocks as within-subject factors were conducted. We used R [version 4.0.4; R Core Team (2021)] for all our analyses. The tidyverse package (Wickham et al., 2019) was used to process the data and visualize them.

\section{Results}

We trained six mice. However, one mouse (D04) consistently chose the left side's stimuli and failed to learn the discrimination task. Therefore, we removed this subject from the subsequent analyses. Figure 4 shows the proportion of correct responses over 90 sessions as a function of 10 -session blocks. The mice learned the discrimination task in all three contexts. However, the mice best discriminated stimuli pairs without a context. A two-way repeated-measures of ANOVA with context type and session block as within-subject factors revealed significant main effects of context $\left(F_{2,8}=36.060\right.$, MSE $\left.=.004, p<.001, \eta g^{2}=.180\right)$ and session blocks $\left(F_{8,32}\right.$ $=10.370$, MSE $\left.=.008, p<.001, \eta g^{2}=.338\right)$. However, the interaction between the context and session blocks was not significant $\left(F_{16,64}=1.353, \mathrm{MSE}=.004, p=.195, \eta g^{2}\right.$ $=.05)$. Post hoc analysis using a Bonferroni correction confirmed significant differences between the no-context and the congruent-context conditions, $p=.003$, as well as between the no-context and the incongruent -context conditions, $p<$ .001 . No difference was found between the congruent and incongruent context, $p=.200$.

Figure 5 shows the mean correct response time over 90 sessions as a function of 10-session blocks. One mouse (D03) responded much slower than other mice, but the response time of this mouse decreased as training proceeded. Other mice showed little change in response times during training. There was no obvious difference in response times across the context type in all mice. A two-way repeated-measures ANOVA with the context type and the session block as within-subject factors indicated no significant main effects of condition $\left(F_{2,8}=1.872, \mathrm{MSE}=78,371.227, p=.215, \eta g^{2}<\right.$ $.001)$ or session block $\left(F_{8,32}=1.134, \mathrm{MSE}=2,461,991.938\right.$, $\left.p=.368, \eta g^{2}=.050\right)$. The interaction between condition and session block was also not significant $\left(F_{16,64}=.783\right.$, MSE $=$ $\left.60,478.561, p=.698, \eta g^{2}=.002\right)$.

\section{Discussion}

Examining the effects of redundant contexts on diagonal direction discrimination is a crucial method for understanding the perceptual mechanisms of emergent properties. This study indicated that discrimination in mice, unlike chimpanzees and humans (Goto, Imura, \& Tomonaga, 2012), was disrupted by redundant contexts. This finding replicated the results of previous studies in rats (Talpos, de-Wit, Olley, Riordan, \& Steckler, 2016) using a within-subject comparison. Moreover, the study investigated another rodent species by including a control context. Notably, mice, unlike rats (Talpos, de-Wit, Olley, Riordan, \& Steckler, 2016), could discriminate diagonal lines embedded within a congruent context. Furthermore, discrimination using an incongruent context was no lower than with a congruent context. Response time showed little difference in the context type and it was thus not a sensitive measure in the present study. Overall, these results suggest that rodents do not perceive the same emergent Gestalts as humans or chimpanzees.

The mice in this study responded to stimuli presented on a touchscreen, and the observation distance might have caused their inability to perceive an emergent Gestalt because, in general, the smaller the stimulus size, the easier it is to attend to configural properties (Goto, Wills, \& Lea, 2004). The 2.6 


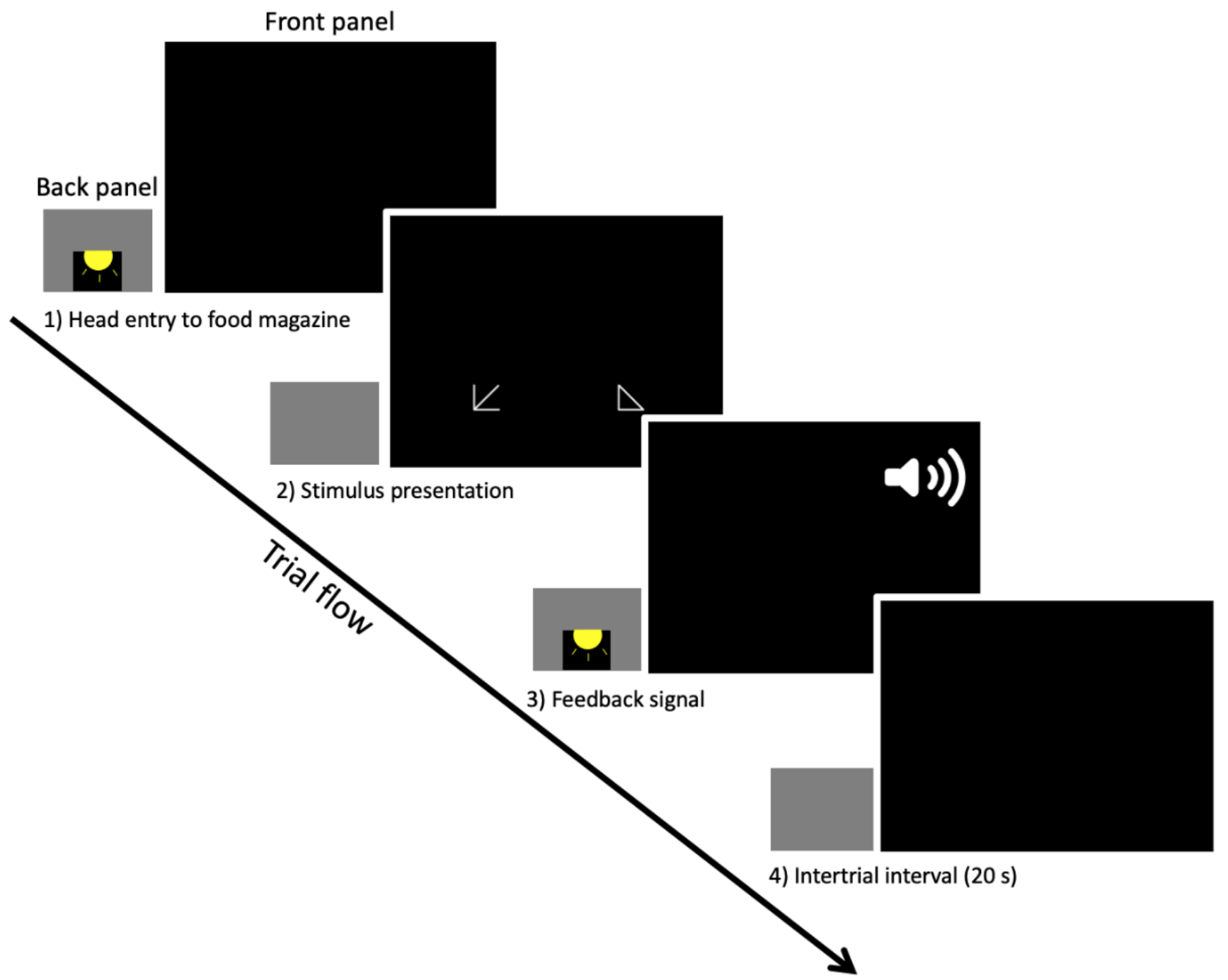

Figure 3. Schematic drawing of the trial sequence for two alternative forced-choice tasks. (1) Each trial started with a head entry to the food magazine when a self-start signal (magazine light blinks at $250 \mathrm{~ms}$ ) was presented. (2) Two images were horizontally aligned on the monitor immediately after the self-start response. (3) When mice made correct responses, they were reinforced by a $20-\mathrm{mg}$ pellet along with 1 -s chime. A 5-s magazine light also signaled the availability of a pellet. A 5-s blackout punished the incorrect response. (4) A 20-s intertrial interval separated trials.

$\mathrm{cm}^{2}$ stimuli were viewed from $30 \mathrm{~cm}$ away, subtending 5 degrees in visual angle in a previous chimpanzee study (Goto, Imura, \& Tomonaga, 2012), whereas the size is much larger for birds (Goto \& Watanabe, 2020). Pigeons viewed $3 \mathrm{~cm}^{2}$ stimuli from $2.5 \mathrm{~cm}$ away, subtending 62 degrees, and crows viewed the identical stimuli from $7 \mathrm{~cm}$ away, subtending 24 degrees. The stimuli used by Talpos, de-Wit, Olley, Riordan, and Steckler (2016) was $4 \mathrm{~cm}^{2}$; however, the viewing distance was unknown. Crijns and Op de Beeck (2019) examined the rat's visual acuity using a similar apparatus to that in Talpos, de-Wit, Olley, Riordan, and Steckler (2016) and estimated that the mean viewing distance was $12.5 \mathrm{~cm}$. This allows us to estimate that the rats' stimulus size was
18 degrees in Talpos, de-Wit, Olley, Riordan, and Steckler (2016). In the present study, the mouse viewed the two stimuli from $2.5-10 \mathrm{~cm}$ away, and thus the stimuli size could be estimated as subtending a visual angle of 57.3-11.4 degrees. Therefore, the inability to perceive emergent Gestalt by birds and rodents might be explained by the larger stimulus size resulting from the visual angle. However, it is unlikely that nonhuman animals fail to see emergent configurations solely due to the observation distance or the stimulus size. For example, Donis and Heinemann (1993), originally reported the disruptive effects of redundant context in pigeons viewing $1 \mathrm{~cm}^{2}$ stimuli at a distance of $8 \mathrm{~cm}$ from the monitor, which subtended 7.2 degrees of visual angle and indicated that the 

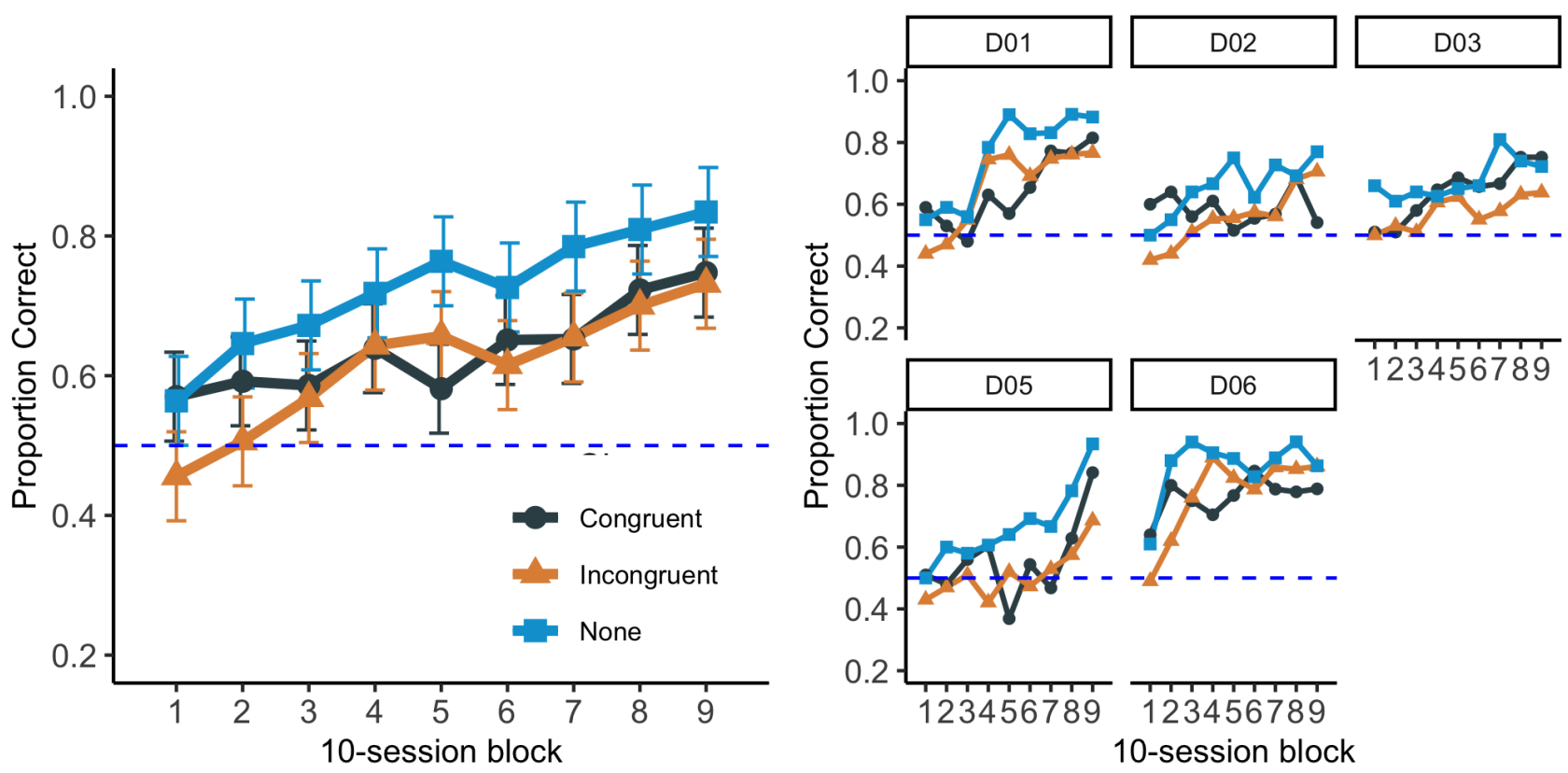

Figure 4. Mean accuracy on three context types throughout 90 sessions. Left. Mean proportion correct of five mice during 90 sessions under each context condition. Error bars delimit 95\% confidential intervals, adjusted for within-subjects error variance (Loftus \& Masson, 1994). Right. Individual data were shown separately.

congruent context disrupted the discrimination of diagonals. No study to date has directly examined the effect of viewing distance or stimuli size, which warrants further investigation.

The differential effect of redundant context among vertebrates is often discussed within the relative dominance of global and local information framework (Lea, Goto, Osthaus, \& Ryan, 2006; Qadri \& Cook, 2015). For example, the relative importance of global and local information is examined using hierarchically constructed figures in two types of configurations (e.g., letters $\mathrm{S}$ or $\mathrm{H}$ presented in small and large sizes). These hierarchical stimuli essentially contain identical information at global (i.e., large figures) and local (i.e., small figures) levels. However, humans discriminate global figures more accurately and quickly than local figures (Navon, 1977). Neiworth, Gleichman, Olinick, and Lamp (2006) demonstrated that 5-year-old children showed a similar global bias as adults when discriminating hierarchically constructed figures in which the elements were arranged densely but not when they were arranged sparsely. In contrast, cotton-top tamarins showed no global bias regardless of the element density. Neiworth et al. (2014) demonstrated that typically developing 3 to 5 -year-old children perceive figures by applying similar Gestalt principles to adults. In contrast, autistic children had difficulties in viewing global aspects of hierarchical stimuli Brosnan, Scott, Fox, and Pye (2004), similar to tamarins, and they also had difficulties in viewing emergent Gestalt, suggesting that their visual sys- tems lack higher-level processing using Gestalt principles. To date, no comparative study has investigated rodents by using hierarchically constructed stimuli. However, Minini and Jefferey (2006) indicated that rats discriminate triangles from squares using luminance differences in the lower hemifield unless those cues become ineffective, implying that local cues have a more vital role in rodents' stimulus control than global cues. Moreover, both Talpos, de-Wit, Olley, Riordan, and Steckler (2016) and our study demonstrated that rodents discriminated diagonals when presented alone than when presented within a redundant context that facilitated discrimination in humans and chimpanzees, suggesting that rodents, similar to tamarins, lack higher-level processing using Gestalt principles.

Previously, it was considered that the differential effect of redundant contexts between primates and birds was due to differences in the two distinctive visual pathways (Goto \& Watanabe, 2020). The same explanation can be applied to the present study of rodents. Most of the retinal ganglion cells (RGC) in primates project to the dorsal lateral geniculate nucleus and the primary visual cortex, whereas only $10 \%$ of primate RGCs project to the superior colliculus Perry, Oehler, \& Cowey (1984). On the other hand, $80 \%$ of mice RGC project to SC (Dreher, Sefton, Ni, \& Nisbett, 1985; Ellis, Gauvain, Sivyer, \& Murphy, 2016; Hofbauer \& Dräger, 1985). Moreover, mice SC neurons are selective to stimulus orientation, suggesting that $\mathrm{SC}$ and its downstream targets 

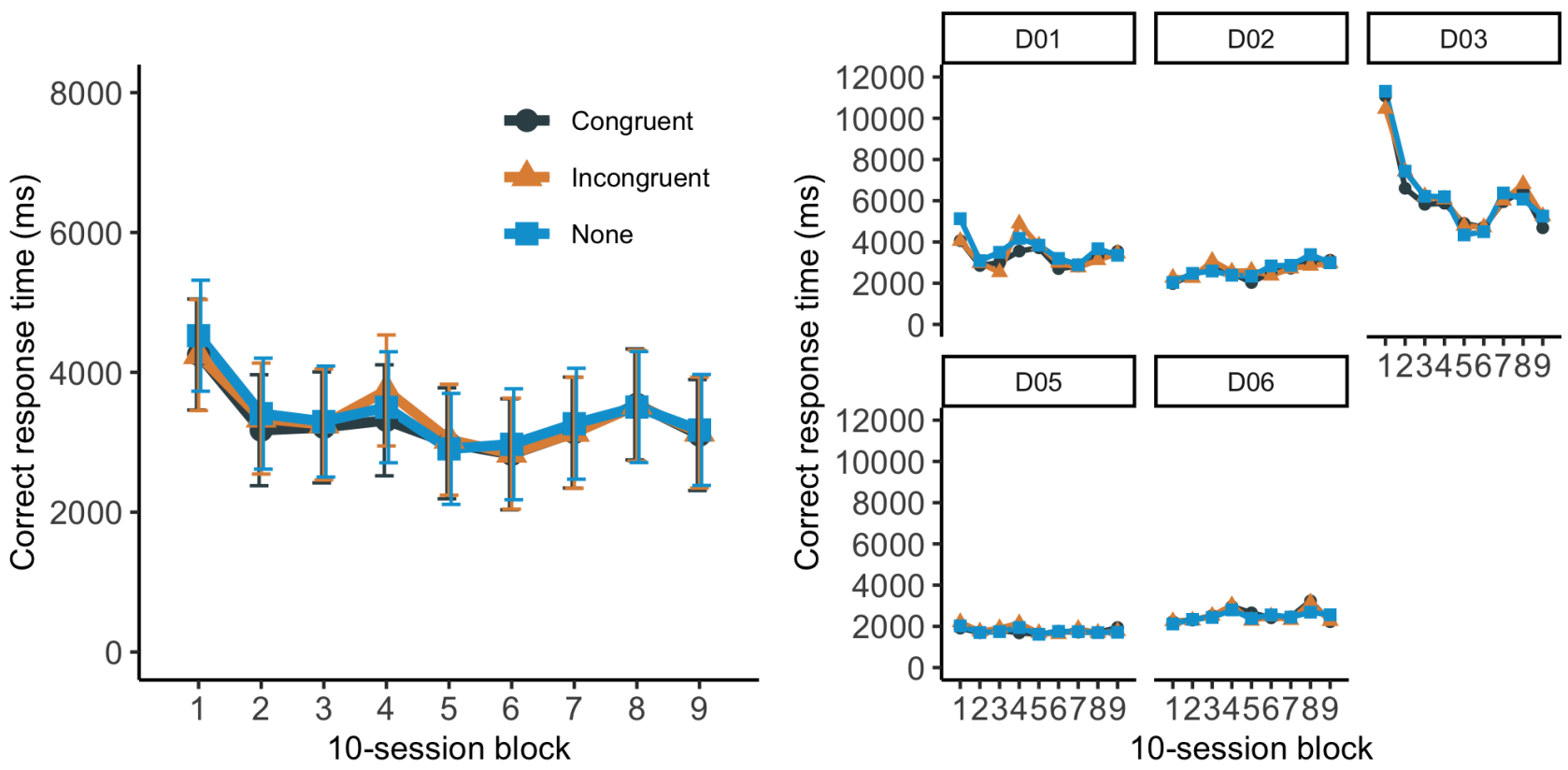

Figure 5. Mean correct-trial response time on three context types throughout 90 sessions. Left. Mean correct response time of five mice during 90 sessions under each context condition. All means were calculated based on log response times and then transformed back to milliseconds by the exponential function. Error bars delimit 95\% confidential intervals, adjusted for within-subjects error variance (Loftus \& Masson, 1994). Right. Individual data were shown separately.

processes object shapes (Feinberg \& Meister, 2015; Wang, Sarnaik, Rangarajan, Liu, \& Cang, 2010; but see, Ahmadlou $\&$ Heimel, 2015). The failure of rodents to perceive emergent Gestalt may also be due to the reliance on the SC pathway because the diagonals' orientation was the discriminative cue in both Talpos, de-Wit, Olley, Riordan, and Steckler (2016) and this study. Whether this failure is limited to the stimulus orientation or also applies to Gestalt principles, in general, is a topic for future research.

The present study's results should be cautiously interpreted because of the narrow scope of the investigation and stimulus manipulation compared to our previous studies with primates and birds (Goto, Imura, \& Tomonaga, 2012; Goto \& Watanabe, 2020). In our previous study, we first trained animals to discriminate $\mathrm{S}+$ from $\mathrm{S}$ - and subsequently, the contingency was reversed, whereas we did not counterbalance the $\mathrm{S}+$ and S- designations in the present study. Moreover, we tested primates and birds with multiple stimulus sets, whereas mice were tested with only a single stimulus set. Both limitations were partially due to the lifespan of mice is shorter than primates and birds. The pellet size is one of the constraints that prevented us from conducting more trials, which precluded us from designing more elaborate and complicated experiments often done with primates and birds. The use of smaller pellets or liquid rewards would give us more flexibility when designing experiments in the future.
Vision is the primary sensory system of many vertebrates; however, comparative studies on phylogenetic differences among species are relatively scarce. Rodents seem to show similar disruptive effects of redundant context as birds. However, why birds and rodents show functional similarities in the perception of emergent Gestalt remain an open question. It might be due to the position of eyes because of the similarity in the disruptive effect of redundant context in birds and rodents. Primates have frontal eyes and high proportions of ipsilateral retinal projection in the optic chiasm, whereas birds and rodents possess laterally situated eyes with few or no ipsilateral projections possessed (Larsson, 2015). Moreover, it remains unknown whether animals with frontal eyes, such as primates and carnivores, perceive emergent Gestalts. Further studies are needed to understand the evolution of Gestalt perceptions. It is suggested that the paradigm used in this study is a useful tool for understanding vertebrates' diversity.

\section{References}

Ahmadlou, M., \& Heimel, J. A. (2015). Preference for concentric orientations in the mouse superior colliculus. Nature Communications, 6(1), 6773. https://doi.org/10. 1038/ncomms 7773

Brosnan, M. J., Scott, F. J., Fox, S., \& Pye, J. (2004). 
Gestalt processing in autism: Failure to process perceptual relationships and the implications for contextual understanding. Journal of Child Psychology and Psychiatry, 45(3), 459-469. https://doi.org/10.1111/j.14697610.2004.00237.x

Crijns, E., \& Op de Beeck, H. (2019). The Visual Acuity of Rats in Touchscreen Setups. Vision, 4(1), 4. https: //doi.org/10.3390/vision4010004

Donis, F. J., Chase, S., \& Heinemann, E. G. (2005). Effects of identical context on visual pattern recognition by pigeons. Animal Learning \& Behavior, 33(1), 90-98. https://doi.org/10.3758/BF03196053

Donis, F. J., \& Heinemann, E. G. (1993). The object-line inferiority effect in pigeons. Perception \& Psychophysics, 53(1), 117-122. https://doi.org/10.3758/BF03211720

Dreher, B., Sefton, A. J., Ni, S. Y., \& Nisbett, G. (1985). The morphology, number, distribution and central projections of Class I retinal ganglion cells in albino and hooded rats. Brain, Behavior and Evolution, 26(1), 1048. https://doi.org/10.1159/000118764

Ellis, E. M., Gauvain, G., Sivyer, B., \& Murphy, G. J. (2016). Shared and distinct retinal input to the mouse superior colliculus and dorsal lateral geniculate nucleus. Journal of Neurophysiology, 116(2), 602-610. https://doi.org/10. 1152/jn.00227.2016

Feinberg, E. H., \& Meister, M. (2015). Orientation columns in the mouse superior colliculus. Nature, 519(7542), 229-232. https://doi.org/10.1038/nature14103

Goto, K., \& Hataji, Y. (2020). Training protocol for touchscreen-based visual discrimination in mice. Japanese Journal of Animal Psychology, 70(1), 1-13. https://doi.org/10.2502/janip.70.1.2

Goto, K., Imura, T., \& Tomonaga, M. (2012). Perception of emergent configurations in humans (Homo sapiens) and chimpanzees (Pan troglodytes). Journal of Experimental Psychology. Animal Behavior Processes, 38(2), 125138. https://doi.org/10.1037/a0026899

Goto, K., \& Watanabe, S. (2020). The whole is equal to the sum of its parts: Pigeons (Columba livia) and crows (Corvus macrorhynchos) do not perceive emergent configurations. Learning \& Behavior, 48(1), 53-65. https: //doi.org/10.3758/s13420-020-00413-w

Goto, K., Wills, A. J., \& Lea, S. E. (2004). Global-feature classification can be acquired more rapidly than localfeature classification in both humans and pigeons. Animal Cognition, 7(2), 109-113.

Hofbauer, A., \& Dräger, U. C. (1985). Depth segregation of retinal ganglion cells projecting to mouse superior col- liculus. The Journal of Comparative Neurology, 234(4), 465-474. https://doi.org/10.1002/cne.902340405

Kelly, D. M., \& Cook, R. G. (2003). Differential effects of visual context on pattern discrimination by pigeons (Columba livia) and humans (Homo sapiens). Journal of Comparative Psychology, 117(2), 200-208. https: //doi.org/10.1037/0735-7036.117.2.200

Larsson, M. L. (2015). Binocular vision, the optic chiasm, and their associations with vertebrate motor behavior. Frontiers in Ecology and Evolution, 3. https: //doi.org/10.3389/fevo.2015.00089

Lea, S. E. G., Goto, K., Osthaus, B., \& Ryan, C. M. E. (2006). The logic of the stimulus. Animal Cognition, 9(4), 247-256. https://doi.org/10.1007/s10071006-0038-3

Loftus, G. R., \& Masson, M. E. J. (1994). Using confidence intervals in within-subject designs. Psychonomic Bulletin \& Review, 1(4), 476-490. https://doi.org/10.3758/ BF03210951

Minini, L., \& Jefferey, K. J. (2006). Do rats use shape to solve "shape discriminations"? Learning \& Memory, 13(3), 287-297. https://doi.org/10.1101//m.84406

Navon, D. (1977). Forest before trees: The precedence of global features in visual perception. Cognitive Psychology, 9(3), 353-383. https://doi.org/10.1016/00100285(77)90012-3

Neiworth, J. J., Gleichman, A. J., Olinick, A. S., \& Lamp, K. E. (2006). Global and local processing in adult humans (Homo sapiens), 5-year-old children (Homo sapiens), and adult cotton-top tamarins (Saguinus oedipus). Journal of Comparative Psychology, 120(4), 323-330. https://doi.org/10.1037/0735-7036.120.4.323

Neiworth, J. J., Whillock, K. M., Kim, S. H., Greenberg, J. R., Jones, K. B., Patel, A. R., ... Kudura, A. G. (2014). Gestalt principle use in college students, children with autism, toddlers (Homo sapiens), and cotton top tamarins (Saguinus oedipus). Journal of Comparative Psychology (Washington, D.C.: 1983), 128(2), 188198. https://doi.org/10.1037/a0034840

Olejnik, S., \& Algina, J. (2003). Generalized Eta and Omega Squared Statistics: Measures of Effect Size for Some Common Research Designs. Psychological Methods, 8(4), 434-447. https://doi.org/10.1037/1082-989X.8.4. 434

Perry, V. H., \& Cowey, A. (1984). Retinal ganglion cells that project to the superior colliculus and pretectum in the macaque monkey. Neuroscience, 12(4), 1125-1137. https://doi.org/10.1016/0306-4522(84)90007-1 
Perry, V. H., Oehler, R., \& Cowey, A. (1984). Retinal ganglion cells that project to the dorsal lateral geniculate nucleus in the macaque monkey. Neuroscience, 12(4), 1101-1123. https://doi.org/10.1016/ 0306-4522(84)90006-X

Pomerantz, J. R. (2003). Wholes, holes, and basic features in vision. Trends in Cognitive Sciences, 7(11), 471-473. https://doi.org/10.1016/j.tics.2003.09.007

Pomerantz, J. R., \& Portillo, M. C. (2011). Grouping and emergent features in vision: Toward a theory of basic Gestalts. Journal of Experimental Psychology. Human Perception and Performance, 37(5), 1331-1349. https: //doi.org/10.1037/a0024330

Pomerantz, J. R., Sager, L. C., \& Stoever, R. J. (1977). Perception of wholes and of their component parts: Some configural superiority effects. Journal of Experimental Psychology: Human Perception and Performance, 3(3), 422-435. https://doi.org/10.1037/0096-1523.3.3.422

Prusky, G. T., West, P. W. R., \& Douglas, R. M. (2000). Behavioral assessment of visual acuity in mice and rats. Vision Research, 40(16), 2201-2209. https://doi.org/10. 1016/S0042-6989(00)00081-X

Qadri, M. A. J., \& Cook, R. G. (2015). Experimental Divergences in the Visual Cognition of Birds and Mammals. Comparative Cognition \& Behavior Reviews, 10, 73-105. https://doi.org/10.3819/ccbr.2015.100004

R Core Team. (2021). R: A language and environment for statistical computing. Vienna, Austria: R Foundation for Statistical Computing. Retrieved from https://www.Rproject.org/

Talpos, J. C., de-Wit, L., Olley, J., Riordan, J., \& Steckler, T. (2016). Do wholes become more than the sum of their parts in the rodent (Rattus Norvegicus) visual system? A test case with the configural superiority effect. The European Journal of Neuroscience, 44(8), 2593-2599. https://doi.org/10.1111/ejn.13350

Wang, L., Sarnaik, R., Rangarajan, K., Liu, X., \& Cang, J. (2010). Visual Receptive Field Properties of Neurons in the Superficial Superior Colliculus of the Mouse. Journal of Neuroscience, 30(49), 16573-16584. https: //doi.org/10.1523/JNEUROSCI.3305-10.2010

Wickham, H., Averick, M., Bryan, J., Chang, W., McGowan, L., François, R., ... Yutani, H. (2019). Welcome to the Tidyverse. Journal of Open Source Software, 4(43), 1686. https://doi.org/10.21105/joss.01686 